\title{
Dog ears: change the approach, not just the name
}

\author{
Adeyinka Molajo \\ Received: 27 November 2010 /Accepted: 2 August 2011 /Published online: 1 September 2011 \\ (C) Springer-Verlag 2011
}

Sir, I read Mashhadi et al.'s [1] communication: 'Dog Earsinappropriate terminology used to describe wound edges' with interest and curiosity.

I agree that calling the resultant raised areas following wound closure 'dog ears' may cause patient distress and dissatisfaction. In the litigious culture in which we practice, we may be leaving ourselves vulnerable to legal action.

Rather than simply changing the way we describe the deformity, I would prefer to seek alternative means of avoiding 'dog ear' formation (or 'topped peak'). I have concentrated on skin lesion excision in this instance.

During my old-fashioned basic surgical training and in my plastic surgery training, I was taught to plan excision by first defining the boundary of the lesion and the necessary excision margins and then design an appropriate ellipse to enable direct closure whilst minimising excess tissue (dog ear or topped peak) formation.

I performed a literature search and discovered two publications which compared elliptical excision and closure with a round excision with the necessary dog ear excision at the primary surgery. Hudson-Peacock et al. [2] showed that by performing circular excision and direct closure, $28 \%$ of lesions could be closed flat without the need for dog ear repair, $38 \%$ of excisions required one dog ear repair and $34 \%$ required two dog ear repairs at time of surgery to achieve a flat closed wound. More significant were the findings that the overall wound lengths were $21 \%$ shorter than if a traditional ellipse was used, and in $45 \%$ of cases, the wound was closed in a different orientation to the original planned elliptical excision.

Seo et al. [3] reported $14 \%$ shorter wounds compared to elliptical excisions and $12 \%$ of wounds being closed flat without need for dog ear repair. Twenty-two percent of wounds were closed in a different orientation to the original plan.

I propose the following approach to skin lesion excision:

1. Define the boundaries of the lesion (using magnification).

2. Determine excision margins required.

3. Draw an appropriate ellipse but not excise ellipse.

4. Excise the lesion and margins.

5. Attempt closure of the round defect and perform dog ear repair as required.

This approach should deal with dog ears, leave a flat and shorter scar and avoid 'chasing of dog ears' associated with traditional wound closure techniques.

\section{References}

1. Mashhadi SA, Loh CYY (2010) 'Dog ear'-an inappropriate terminology to describe wound edges. Eur J Plast Surg 33:381

2. Hudson-Peacock MJ, Lawrence CM (1995) Comparison of wound closure by means of dog ear repair and elliptical excision. J Am Acad Dermatol 32(4):627-630

3. Seo SH, Son SW, Kim IH (2008) Round excisions lead to shorter scars and better scar positioning than traditional elliptical excisions. Dermatology 217(3):276-280 